\title{
Role of oral semaglutide in management of type 2 diabetes
}

\author{
Nasser Mikhail* \\ David-Geffen School of Medicine, Endocrinology Division, Olive-View-UCLA Medical Center, USA
}

\begin{abstract}
Objective: To review efficacy and safety of the first orally available glucagon-like peptide (GLP-1) receptor agonist semaglutide.

Methods: PubMed search published in English, French and Spanish from January 2000 until September 4, 2019. Search terms included "oral semaglutide”, "semaglutide”, glucagon-like peptide-1 receptor, "clinical trials”, “absorption”, "metabolism”, “efficacy”, "safety, "cardiovascular” 'kidney disease”. Randomized trials, review articles, expert opinions and editorials are included in the review.

Results: Oral semaglutide is effectively absorbed in the stomach by absorption enhancer but has to be taken in the fasting state with water, and no food allowed for 30 min after intake. It is generally comparable in efficacy to the subcutaneous form of semaglutide. When compared to liraglutide, oral semaglutide is slightly superior in decreasing hemoglobin A1c (HbA1c) (-0.3\% vs liraglutide) and weight ( $-1.3 \mathrm{~kg}$ vs liraglutide) but is associated with more frequent adverse effects (reported by $80 \%$ vs $74 \%$ of patients). Limited data suggest that oral semaglutide is safe and effective in patients with moderate degree of renal impairment. A large randomized trial of median follow-up of 15.9 months, showed that oral semaglutide was non-inferior to placebo in terms of cardiovascular events and mortality, and might have beneficial effects on reducing some of these events.

Conclusions: Oral semaglutide has an efficacy and safety profile consistent with the class of GLP-1 receptor agonists. It represents a useful therapeutic option for patients with type 2 diabetes who are reluctant to take injections. Further studies are needed to establish its long-term efficacy and safety in a large population of type 2 diabetes, including those with chronic kidney disease.
\end{abstract}

\section{Introduction}

Oral semaglutide is the only orally available GLP-1 receptor agonist. To achieve adequate bioavailability after oral administration, co-formulation with an absorption enhancer is needed. Thus, oral semaglutide is being developed as a co-formulation with the absorption enhancer sodium $\mathrm{N}$-[8-(2-hydroxybenzoyl) amino caprylate] (SNAC) [1]. The mode of action of the latter enhancer involves a localized increase in $\mathrm{pH}$ that protects semaglutide against proteolytic degradation and facilitates its absorption across the gastric epithelium [1]. Oral semaglutide should be taken in the fasting state because food may hinder its absorption from the stomach [1]. After intake, time to maximum plasma concentration ranges between 0.38 and $0.7 \mathrm{~h} \mathrm{[2]}$. Plasma half-life of oral semaglutide is approximately 1week, which is similar to semaglutide administered subcutaneously [2].

\section{Efficacy of oral semaglutide}

\section{Comparison with subcutaneous weekly semaglutide}

The efficacy of oral semaglutide is dose-dependent, and seems inferior to the subcutaneous formulation of semaglutide when used in doses lower than $20 \mathrm{mg} / \mathrm{d}$. Thus, in one phase 2 randomized trial, reduction in levels of haemoglobin $\mathrm{A} 1 \mathrm{c}$ (HbA1c) with oral semaglutide at daily doses of $10 \mathrm{mg}$ was $1.2 \%$ vs placebo, whereas the corresponding decrease with subcutaneous semaglutide ( $1.0 \mathrm{mg}$ weekly) was $-1.6 \%$ [3]. Meanwhile, the efficacy of the $20 \mathrm{mg}$-dose of oral semaglutide was not statistically different from the subcutaneous form of semaglutide ( $-1.4 \%$ and $-1.6 \%$ vs placebo, respectively) [3]. Unfortunately, in the latter study, the currently recommended 14-mg dose of oral semaglutide was not used [3].

\section{Comparison with other GLP-1 agonists}

In a randomized, double-blind and double-dummy trial, oral semaglutide was compared to the widely used GLP-1 receptor agonist liraglutide (given subcutaneously once daily) and placebo [4]. After 52 weeks, $\mathrm{HbAlc}$ reduction in the semaglutide group was $0.3 \%$ greater than liraglutide, and $1.0 \%$ greater than in placebo group [4]. From a mean $\mathrm{HbAlc}$ levels of $8.0 \%$, proportions of patients who reached $\mathrm{HbAlc}<7.0 \%$ were $60.7 \%$ and $55 \%$ in the oral semaglutide and liraglutide group, respectively $(p=0.11)$ [4].

\section{Comparison with sitagliptin}

Oral semaglutide was shown to be more effective than sitagliptin $100 \mathrm{mg} / \mathrm{d}$ in another randomized, double-blind, double dummy trial including patients with type 2 diabetes uncontrolled on metformin \pm sulfonylurea (SU) [5]. After 26 weeks, HbA1c reduction from baseline was $-1.0 \%,-1.3 \%$, and $-0.8 \%$ with oral semaglutide $7 \mathrm{mg} / \mathrm{d}, 14 \mathrm{mg} / \mathrm{d}$, and sitagliptin $100 \mathrm{mg} / \mathrm{d}$, respectively [5].

\section{Effect of oral semaglutide on body weight}

Similar to other members of GLP-1 receptor agonists, oral semaglutide consistently induce weight loss close to $4 \mathrm{~kg}$ compared

${ }^{\star}$ Correspondence to: Nasser Mikhail, David-Geffen School of Medicine, Endocrinology Division, Olive-View-UCLA Medical Centre, 14445 Olive View Drive, Sylmar, CA, 91342, USA, E-mail: nmikhail@dhs.lacounty.gov

Key words: oral semaglutide, absorption enhancer, GLP-1 receptor agonist, efficacy, safety, renal impairment

Received: September 02, 2019; Accepted: September 16, 2019; Published: September 19, 2019 
to placebo after 52 weeks [4]. In the largest randomized trial of oral semaglutide, mean reduction in weight at 62 weeks compared with baseline was $4.2 \mathrm{~kg}$ in the oral semaglutide group and $0.8 \mathrm{~kg}$ in the placebo group (statistical significance was not reported) [6]. Inspection of weight curves showed that maximum weight reduction was attained between 26 and 38 weeks followed by rebound [6].

\section{Use of oral semaglutide in chronic kidney disease}

The pharmacokinetics of oral semaglutide were not affected in subjects with renal impairment [7]. In fact, semaglutide is metabolized by proteolytic cleavage, and not cleared by specific organ [7]. In one randomnized, double-blind, placebo-controlled trial of 26 weekduration, oral semaglutide was effective in patients with advanced type 2 diabetes and stage 3 chronic kidney disease defined as estimated glomerular filtration rate (eGFR) between $30-59 \mathrm{ml} / \mathrm{min} / 1.73 \mathrm{~m}^{2}$ [8]. The reduction in $\mathrm{HbAlc}$ value was $1.0 \%$ compared to baseline and $0.8 \%$ compared with placebo [8]. In addition, mean reduction in weight was $3.4 \mathrm{~kg}$ and $0.9 \mathrm{~kg}$ in the oral semaglutide and placebo, respectively with a significant difference of $2.5 \mathrm{~kg}$ [8]. However, oral semaglutide seems less well tolerated in patients with chronic kidney disease (CKD) Hence, $15 \%$ discontinued oral semaglutide due to adverse effects compared with $5 \%$ in placebo [8]. Overall, renal function as reflected by eGFR was unchanged throughout the trial in both treatment groups [8]. Clearly, more studies are needed with longer duration of followup to establish renal safety of oral semaglutide in patients with various degrees of CKD.

\section{Effect of oral semaglutide on cardiovascular outcomes}

PIONEER 6 is the largest randomized trial available for the evaluation of cardiovascular (CV) safety of oral semaglutide [6]. The main purpose of the PIONEER 6 trial is to test whether oral semaglutide is non-inferior to placebo in terms of CV outcomes in patients with type 2 diabetes and high CV risk at baseline. An overview of trial design is summarized in table 1 . After a median follow-up of

Table 1. Overview and main results of PIONEER 6 [6]

\begin{tabular}{|c|c|}
\hline Design & Randomized, double-blind, multi-center, 2 groups \\
\hline Patients & $\begin{array}{l}\mathrm{n}=3183 \text { with type } 2 \text { diabetes, mean age } 66 \mathrm{y} / \mathrm{o}, 31 \% \\
\text { females, } 85 \% \text { had established cardiovascular disease, } \\
\text { baseline HbA1c } 8.2 \%\end{array}$ \\
\hline Main outcome & $\begin{array}{l}\text { Time to first occurrence of cardiovascular death, non-fatal } \\
\text { myocardial infarction, or non-fatal stroke }\end{array}$ \\
\hline Median follow-up & 15.9 months \\
\hline $\begin{array}{l}\text { Concomitant diabetes } \\
\text { medications at baseline }\end{array}$ & $\begin{array}{l}\text { Metformin } 77 \% \text {, insulin } 60 \% \text {, Sulfonylureas } 32 \% \text {, Sodium- } \\
\text { glucose cotransporter- } 2 \text { inhibitors } 10 \%\end{array}$ \\
\hline Intervention & Oral semaglutide $14 \mathrm{mg} / \mathrm{d}(\mathrm{n}=1591)$, placebo $(\mathrm{n}=1592)$ \\
\hline $\begin{array}{l}\text { Number of events of } \\
\text { primary outcome }\end{array}$ & $\begin{array}{l}\text { Semaglutide } 61(3.8 \%) \text {, placebo } 76(4.8 \%), \mathrm{HR}^{*} 0.79 \\
(95 \% \text { CI } 0,57-1.11)\end{array}$ \\
\hline Death from any cause & $\begin{array}{l}\text { Semaglutide } 23(1.4 \%) \text {, placebo } 45(2.8 \%) \text {, HR } 0.51(95 \% \\
\text { CI } 0.31-0.84)\end{array}$ \\
\hline $\begin{array}{l}\text { Mean change in weight } \\
\text { compared with baseline }\end{array}$ & Semaglutide $-4.2 \mathrm{~kg}$, placebo $-0.8 \mathrm{~kg}$ \\
\hline $\begin{array}{l}\text { Mean change in HbA1c } \\
\text { compared with baseline }\end{array}$ & Semaglutide $-1.0 \%$, placebo $-0.3 \%$ \\
\hline $\begin{array}{l}\text { Patients with severe } \\
\text { hypoglycemia }\end{array}$ & Semaglutide $23(1.4 \%)$, placebo $13(0.8 \%)$ \\
\hline $\begin{array}{l}\text { Discontinuation of study } \\
\text { drug due to adverse effects }\end{array}$ & Semaglutide $11.6 \%$, Placebo $6.5 \%$ \\
\hline
\end{tabular}

*HR: Hazard Ratio. Difference in primary outcome was statistically significant $(p<0.001)$ for non-inferiority of oral semaglutide compared to placebo, but non-significant $(p=0.17)$ with respect to superiority of semaglutide over placebo.
15.9 months, the primary outcome composed of CV death, nonfatal myocardial infarction (MI) and nonfatal stroke occurred in $3.8 \%$ of patients randomized to oral semaglutide and $4.8 \%$ of those randomized to placebo corresponding to $21 \%$ risk reduction (hazard ratio $0.79,95 \%$ CI, $0.57-1.11), p<0.001$ for non-inferiority, but $p=0.17$ for superiority [6].

In addition, oral semaglutide was associated with decreased risk in death from any cause ( $1.4 \%$ vs $2.4 \%$ placebo, hazard ratio $0.51,95 \% \mathrm{CI}$ $0.31-0.84)$, and death from CV causes (0.9\% vs $1.9 \%$ placebo, hazard ratio $0.49,95 \%$ CI $0.27-0.92$ ), There was a non-significant trend in risk reduction in the semaglutide group in nonfatal stroke and in heart failure requiring hospitalization, and inverse trend toward increase risk of nonfatal MI and unstable angina requiring hospitalization [6]. Nevertheless, these data provide reassurance about short-term CV safety of oral semaglutide and are in line with results of CV trials of other members of GLP-1 receptor agonists $[9,10]$, including subcutaneous semaglutide [11].

\section{Safety of oral semaglutide}

In general, the safety profile of oral semaglutide is consistent with the class of GLP-1 receptor agonists. Indeed, gastrointestinal (GI) adverse effects, particularly nausea, are the most common adverse effect reported by $20 \%$ of patients receiving oral semaglutide compared with $4 \%$ with placebo [4]. Moreover, GI adverse effects were the commonest cause of permanent drug discontinuation in $11.6 \%$ of patients randomized to oral semaglutide compared to $6.5 \%$ of those randomized to placebo [6].

In an attempt to predict tolerance of oral semaglutide in clinical practice, one randomized open-label trial used flexible dose of oral semaglutide $(\mathrm{n}=253)$ versus sitagliptin $100 \mathrm{mg} / \mathrm{d}(\mathrm{n}=250)$ [12]. At week 52 , of the 212 patients on semaglutide, $41 \%$ could not tolerate the maximum dose $(14 \mathrm{mg} / \mathrm{d})$ due to nausea and vomiting and were receiving either the $7 \mathrm{mg}$-dose (30\%) or the $3 \mathrm{mg}$-dose (9\%) [12].

Severe hypoglycaemia requiring assistance of another person occurred in $1.4 \%$ and $0.8 \%$ of patients receiving oral semaglutide and placebo, respectively. All severe hypoglycaemic events occurred in patients taking concomitant insulin or SU [6].

Worsening of diabetic retinopathy was previously reported in a large trial of subcutaneous semaglutide occurring in 3.0\% of subjects compared with $1.8 \%$ of patients receiving placebo, hazard ratio, 1.76, 95\% CI 1.11-2.78, $p=0.02$ ) [11]. In PIONEER 6, the proportions of patients with adverse effects related to retinopathy were numerically greater with oral semaglutide, $7.1 \%$ vs $6.3 \%$ with placebo [6]. However, patients with baseline diabetic retinopathy were excluded [6]. One long term trial specifically designed to investigate the relationship between subcutaneous semaglutide, and retinopathy is underway and should further clarify this issue.

\section{Who is the best candidate for oral semaglutide?}

Based on the drug characteristics and available data discussed above, oral semaglutide may be appropriate add-on second- or thirdline drug for patients with type 2 diabetes uncontrolled on metformin and other oral agents who are overweight, and reluctant to take any form of injections. In addition, oral semaglutide may be useful for patients prone for hypoglycaemia and those with moderate renal impairment.

Patients with proliferative retinopathy should not use any form of semaglutide until more safety data becomes available. Advantages and limitations of oral semaglutide are summarized in table 2. 
Table 2. Advantages and limitations of oral semaglutide

\begin{tabular}{|l|l|}
\hline Advantages & Limitations \\
\hline Taken orally once daily & $\begin{array}{l}\text { Has to be taken on empty stomach with water, } \\
\text { and not to take any drink or food or other oral } \\
\text { medications for at least 30 min after. }\end{array}$ \\
\hline $\begin{array}{l}\text { Comparable efficacy to } \\
\text { subcutaneous semaglutide and } \\
\text { liraglutide }\end{array}$ & $\begin{array}{l}\text { Common GI adverse effects (10-15\% vs 2-5\% with } \\
\text { placebo) }\end{array}$ \\
\hline $\begin{array}{l}\text { Causes moderate weight loss, } \\
\text { approximately } 3-4 \text { kg after 1 year }\end{array}$ & $\begin{array}{l}\text { Takes } 8 \text { weeks of dose escalation to reach maximum } \\
\text { dose }(14 \mathrm{mg} / \text { d) leading to delay to reach maximum } \\
\text { efficacy }\end{array}$ \\
\hline $\begin{array}{l}\text { Low risk of hypoglycemia, except } \\
\text { when used with insulin or SU }\end{array}$ & $\begin{array}{l}\text { Its long-term safety not established, particularly its } \\
\text { effect on worsening retinopathy }\end{array}$ \\
\hline $\begin{array}{l}\text { May be used in moderate renal } \\
\text { impairment (eGFR 30-59 ml/ } \\
\text { min/1.73 } \mathrm{m}^{2}\end{array}$ & \\
\hline
\end{tabular}

\section{Conclusions and future needs}

No doubt, oral semaglutide provides a practical treatment option for patients with type 2 diabetes. This oral formulation is as effective as the subcutaneous form of semaglutide and at least as effective as liraglutide. The drug profile of oral semaglutide closely mimics other drugs pertaining to the class of GLP-1 agonists including mild weight loss, decrease risk of hypoglycemia, possible decrease in CV events, but increase in GI adverse effects. Randomized trials of adequate power are needed to establish long-term efficacy and safety of oral semaglutide in a broader population of patients with type 2 diabetes having different degrees of CV risk factors and renal function at baseline. These trials should carefully examine safety issues of particular concern such as worsening of diabetic retinopathy.

\section{Conflict of interest}

The author has no conflict of interest to declare.

\section{References}

1. Buckley S, Baekdal TA, Vegge A, Maarbjerg SJ, Pyke C, et al (2018) Transcellular stomach absorption of a derivatized glucagon-llike peptide-1 receptor agonist. Sci Transl Med 10: eaar 7047: 1-13. [Crossref]
2. Granhall C, Donsmark M, Blicher TM, et al (2019) Safety and pharmacokinetics of single and multiple ascending doses of the novel oral human GLP-1 analogue, semaglutide, in healthy subjects and subjects with type 2 diabetes. Clin Pharmacokinet 58: 761-791. [Crossref]

3. Davies M, Pieber TR, Hartfot-Nielson M, Hansen OH, Jabbour S, Rosenstock J (2017) Effect of oral semaglutide compared with placebo and subcutaneous semaglutide on glycemic control in patients with type 2 diabetes. A randomized clinical trial. JAMA 318: 1460-1470. [Crossref]

4. Prately R, Amod A, Hoff ST, Kadowaki T, Lingvay I, et al (2019) Oral semaglutide versus subcutaneous liraglutide and placebo in type 2 diabetes (PIONEER 4): a randomised, double-blind, phase 3a trial. Lancet 394: 39-50. [Crossref]

5. Rosenstock J, Allison D, Birkenfeld AL, Blicher TL, Deenadayalan S, Jacobsen JB, et al. (2019) Effect of additional oral semaglutide vs sitagliptin on glycated hemoglobin in adults with type 2 diabetes uncontrolled with metformin alone or with sulfonylurea The PIONEER 3 randomized clinical trial. JAMA 321: 146-1480. [Crossref]

6. Husain M, Birkenfeld AL, Donsmark M, Dungan K, Eliaschewitz M, et al (2019) Oral semaglutide and cardiovascular outcomes in patients with type 2 Diabetes. $N$ Eng $J$ Med 381: 841-851. [Crossref]

7. Granhall C, Sondergaard FL, Thomsen M, Anderson TW (2018) Pharmacokinetics, safety and tolerability of oral semaglutide in subjects with renal impairment. Clin Pharmacokinet 57: 1571-1580. [Crossref]

8. Mosenzon O, Blicher TM, Rosenlad S, Erisksson JW, Heller S, et al (2019) Efficacy and safety of oral semaglutide in patients with type 2 diabetes and moderate renal impairment (PIONEER 5): a placebo-controlled, randomised phase 3a trial. Lancet Diab Endocrinol 7: 515-527. [Crossref]

9. Marso SP, Daniels GH, Brown-Frandsen K, Kristensen P, et al. (2016) LEADER Steering Committee: LEADER Trial Investigators (2016). Liraglutide and cardiovascular outcomes in type 2 diabetes. $N$ Eng J Med 375: 311-322. [Crossref]

10. Gerstein HC, Colhun HM, Dagenais HR, Diaz R, Lakshmanan M, et al. (2019) Dulaglutide and cardiovascular outcomes in type 2 diabetes (REWIND): a doubleblind, randomized placebo-controlled trial. Lancet 394: 121-130. [Crossref]

11. Marso S, Bain SC, Consoli A, Eliaschewitz FG, Jodar E, et al. (2016) Semaglutide and cardiovascular events in patients with type 2 diabetes. $N$ Engl J Med 375: 1834-1844. [Crossref]

12. Pieber TR, Bode B, Mertens A, Cho YM, Christiansan E, et al. (2019) Efficacy and safety of oral semaglutide with flexible dose adjustment versus sitagliptin in type 2 diabetes (PIONEER 7): a multicenter, open-label, randomized, phase 3a trial. Lancet Diab Endocrinol 7: 528-539. [Crossref]

Copyright: (2019 Mikhail N. This is an open-access article distributed under the terms of the Creative Commons Attribution License, which permits unrestricted use, distribution, and reproduction in any medium, provided the original author and source are credited. 